\title{
Supporting Transitions with Creative Software Engineering
}

\author{
Clare J. Hooper \\ University of Southampton \\ Southampton, SO17 1BJ, UK \\ clare@ecs.soton.ac.uk
}

\author{
David E. Millard \\ University of Southampton \\ Southampton, SO17 1BJ, UK \\ dem@ecs.soton.ac.uk
}

\begin{abstract}
In this paper we explore the use of creative software engineering design methods, particularly for rebuilding experiences in new contexts. We present results from a comparative evaluation of three approaches (scenarios with personas, TAPT, and no formal method): these suggest that techniques such as brainstorming are helpful, while structure and formality can distract from the creative process. Scenarios' user-focus was noted as positive, as were TAPT's analytical output and abstraction of experiences away from their original contexts. The best approach to design may be to apply multiple methods in a complementary fashion.
\end{abstract}

Context, design, evaluation, Scenarios, TAPT, transitions, user experience

\section{INTRODUCTION}

Modern software systems are increasingly fluid and multimodal, with user interactions occurring across a variety of devices and infrastructures (desktop, web, mobile, embedded systems, etc.). This complexity of potential interaction means that understanding user experience (UX) is an increasingly difficult part of the software design process - for example, how does online retail link with bricks-and-mortar shopping, how can we make social networking systems more accessible for the elderly, can web 2.0 style public collaboration work in the real world as well as the on the web?

In this paper we provide an overview of creative software engineering methods for understanding UX and discuss results of a comparative evaluation of two methods, particularly with regard to understanding the methods' creative qualities.

\section{CREATIVE SOFTWARE ENGINEERING}

Computer science as a field certainly encompasses creative elements (Titchener, 2005), and there exist various creative methods for gaining insight into users and their perspectives (Maiden, 2004). One is cultural probes (Gaver, 1999), packages of maps, postcards and other materials, designed to elicit open, inspirational responses from people with whom computer scientists will work.

Another method for understanding users is personas (Grudin, 2002), the building of 'actors' or detailed descriptions of people representative of user groups. They might include name, age, appearance, clothes, occupation, family, friends, pets, gender and ethnicity, and are often based on real data. Scenarios (Tedjasaputra, 2004) are broader narratives that describe different situations in which technology is used. They are stories, with setting, characters, and plot. They help designers and analysts find assumptions they have made, and can be used in conjunction with personas.

Probes, scenarios and personas focus on end users, but it is not always clear how they scaffold an understanding of UX grounded in specific contexts. TAPT is a new technique which addresses this (Hooper, 2010). It involves a structured analysis of experiences, sufficient to support their transition to new contexts. E.g., the design of physical systems inspired by online experiences (e.g. wiki-based collaborative areas in museums) and web-based interactions based on physical experiences (e.g. a web-based picnic).

\section{COMPARATIVE EVALUATION}

In late 2009, we conducted a comparative evaluation of scenarios/personas and TAPT as creative design methods. In the evaluation, 43 software engineers worked in small groups on varying design tasks using varying methods. Full details are in (Hooper, 2010).

Scenarios with personas were chosen as representative of current design practice. We also included No Method as a neutral baseline against which the other approaches could be judged (No 
Method was presented to participants as a verbal discussion during the design process). The tasks involved re-imagining experiences in new contexts, for example re-providing microblogging in a care home for the elderly.

Participants reported on how well the methods supported creation of imaginative, novel designs. Regarding TAPT, participants were positive about the structured analysis ("Gave an easy-to-refer to sheet to bring ideas into focus"; "With all the huge components identified, was easy to link them together in a new way"), focus ("It helped us concentrate on the parts we needed to design creatively"), and helpfulness of abstracting the original experience ("Separates technology from effect"; "The distilled experience forced a more abstracted idea, which helped reshape the experience much more easily"). Negative comments concerned rigidity ("constrained by need to complete specific boxes").

Comments on Scenarios were positive about the user-focus ("You try to envision yourself as these people"; "Personas ... lead to good ideas") and brainstorming ("Being able to brainstorm lets you be imaginative"). Some comments concerned the need for a balance between freedom and structure ("Discussion/brainstorming were good for creativity, but needed a clear structure later"), while others disliked the rigidity ("it's so prescriptive, your brain is in the habit of following instructions. I think that made us less imaginative"). Another participant commented that there was no real creative leap ("The design came incrementally... so although it is very tailored to the users, it isn't exactly 'novel'.").

Comments on No Method were positive about the freedom ("Lack of direction meant we discussed many ideas - plausible or not!"; "we were able to be as creative as we chose"), and lack of structure ("it made us concentrate on ideas rather than outputting documents"; "I prefer less structured methods"). The ease of use was praised ("It was easy to throw around any ideas that occurred"), although there was concern about scalability ("In larger groups [than 2], some opinions may get drowned out"). Other participants commented that the method was only as creative as its users ("Had a neutral effect. Didn't hinder and didn't help."; "the method was only ever as imaginative as we are").

Participants also rated methods' support in creating imaginative and novel designs on a Likert-scale. Interestingly, No Method was rated highly $(82 \%$ of users said it did 'well' or 'very well'), compared to Scenarios and TAPT (63\% and 58\% respectively).

\section{DISCUSSION}

We tested these methods for transitioning between different contexts of interaction, both physical and digital. Each method appears to have strengths and weaknesses in this context: participants liked the structured analysis, focus and abstraction of TAPT, although they disliked its rigid structure (mirroring similar comments about Scenarios). Positive comments on Scenarios included its user-focus, while positive comments on No Method centred on its free, unstructured, easy-to-apply nature: negative comments on this method concerned the lack of support for creativity.

The high rating of No Method's support for creativity is interesting, and perhaps results from people's dislike of the structured, procedural nature of the other two methods in the context of being free to come up with ideas. Although participants thought No Method allowed them to be more creative, it would appear that TAPT and Scenarios enabled the production of creative results with certain other advantages, as detailed above. Overall, it seems likely that participants' valuejudgements about their designs will have been affected by the methods used. We plan a blind review of the design artefacts to gain objective insight into the methods' efficacy.

We can conclude that all three methods support, to some extent, the creation of imaginative and novel designs which involve transitions between contexts of interaction. Each method clearly has certain strengths and weaknesses: No Method seems to be an ideal starting point in the design process, perhaps to be followed by the application of Scenarios towards identifying user groups, and then the application of TAPT to elicit the explicit experiences of each user group.

\section{REFERENCES}

Gaver, B., Dunne, T.,and Pascenti, E. (1999) Design: Cultural probes. Interactions, 6, 21-29. Grudin, J., Pruitt, J. (2002) Personas, participatory design and product development. Participatory Design, Palo Alto. 144-161.

Hooper, C.J., Millard, D.E. (2010). Teasing Apart and Piecing Together: Towards Understanding Web-based Interactions. Web Science. NC, USA. Maiden, N., Gizikis, A., Robertson, S. (2004) Provoking Creativity: Imagine What Your Requirements Could be Like. IEEE Software, 21, 68-75.

Tedjasaputra, A., Sari, E., Strom, G. (2004) Sharing and Learning through Pair Writing of Scenarios. NordiCHI. Tampere, Finland. Titchener, C. (2005) Reviewing the Arts. Lawrence Erlbaum Associations. 